\title{
Simulation of deformation and fracture processes in nanocomposites
}

\author{
A.V. Vakhrushev, A.Y. Fedotov \\ Udmurt Federal Research Center of the Ural Branch of the Russian Academy of Sciences, Izhevsk 426067, Russian Federation \\ Kalashnikov Izhevsk State Technical University, Izhevsk 426069, Russian Federation \\ vakbrushev-a@yandex.ru, bttp://orcid.org/0000-0001-7901-8745 \\ aleafed@gmail.com, bttp://orcid.org/0000-0002-0463-3089
}

\begin{abstract}
The paper studies the processes of deformation and fracture in nanocomposites. The study was carried out by the method of mathematical modeling. The behavior of the nanosystem was described by the molecular dynamics apparatus. A modified immersed atom method was used as a potential. Demonstrated theoretical approaches to the study of the mechanical properties of nanocosposites and the processes of their failure. Formulas for calculating the stress, strain tensors and displacement were given. To maintain a constant temperature in the nanosystem, a Nose Hoover thermostat was used. The failure of nanocomposites was considered in the process of tension and shear deformation. Pure aluminum, a composite with an aluminum matrix and a filler in the form of spherical iron particles, and a composite with an aluminum matrix and a filler in the form of a cylindrical iron fiber were used as samples. After the filler was introduced into the nanocomposite, the sample was relaxed to ensure its more stable state. The simulation allowed us to establish the basic laws of changes in the atomic structure of the matrix and nanocomposite fillers during deformation and fracture. It is shown that the processes of deformation and failure of nanocomposites substantially depend both on the structure and types of loading of the material. The results of the research can be used to study the processes of deformation of nanocomposite materials with promising functional properties.
\end{abstract}

KEYwORDS. Nanostructures; Nanocomposites; Molecular dynamics; Deformation; Rupture.

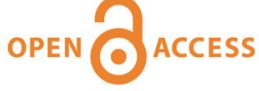

Citation: Vakhrushev, A.V., Fedotov, A.Y., Simulation of deformation and fracture processes in nanocomposites, Frattura ed Integrità Strutturale, 49 (2019) 370-382.

Received: 24.04 .2019

Accepted: 30.05 .2019

Published: 01.07.2019

Copyright: (C) 2019 This is an open access article under the terms of the CC-BY 4.0, which permits unrestricted use, distribution, and reproduction in any medium, provided the original author and source are credited.

\section{INTRODUCTION}

anocomposites are widely used due to their special properties, which better distinguish them from ordinary materials. Increased strength and wear resistance, optical and semiconductor characteristics make these materials promising for operation in various fields of science and technology [1-3]. 
The introduction of nanocomposites requires their detailed study at various scale levels. Mathematical modeling is, in this case, a promising method for studying the fundamental laws of their formation, deformation and failure. The theoretical approach reduces the cost of conducting expensive natural experiments. Mathematical modeling also allows us to discover and predict the potentially new and promising properties of these materials [4-7].

Many physical characteristics of nanoobjects, such as thermal conductivity coefficient [8, 9], diffusion coefficient [10, 11] and electrical conductivity $[12,13]$, belong to macroparameters. Strain and stress tensors occupy a special place, since these values determine the deformation and failure of nanomaterials [14-16]. When calculating these values, the problem of consistency of scale levels arises when, due to the physical meaning of the parameters under consideration, or for other reasons, there is no uniquely defined methodology for determining them. In connection with the current situation, the question of describing macrocharacteristics at the nanoscale level, including their mechanical features, remains relevant. The operation of cryogenic mechanical system for the study of nano- and microscale behavior of materials at low temperatures is designed and investigated in the publication [17]. The experiments were carried out under the supervision of a scanning electron microscope, which was equipped with a module with simultaneous cooling of the sample subjected to uniaxial compression. Samples with diameters from 400 to $1300 \mathrm{~nm}$ made on the basis of Nb and W metals showed higher values of yield strength and greater deformations at lower temperatures. Comparison of characteristics was carried out with similar values obtained at temperatures close to normal. The authors explain these differences by nanoscale plasticity and internal resistance of the atomic crystal lattice of the sample material. The results obtained can be used in such materials as topological insulators [18].

A review of theoretical models of plastic deformation processes in crystalline nanomaterials is given in [19]. Two-phase nanocomposites with internal boundaries and grain boundaries along component phases are considered as target materials. Special attention is paid to the behavior of various mechanisms of plastic deformation depending on transitions between materials and on the grain size. Physical mechanisms in nanocomposites, such as sliding along the grain boundaries of a material, movement of crystal lattice dislocations, and processes of diffusion plasticity, are illustrated.

The topical are the studies of nanomaterials based on supercarbon nanotubes. The authors of [20] analyzed the radialdeformation capabilities of super-carbon nanotubes under uniaxial tension using the method of molecular-dynamic modeling. The results obtained in this work demonstrate good reversible and adjustable parameters of the radial shrinkage of nanotubes, which may be due to their hierarchical structure. Depending on the topology in the areas of the nanotube junction, the levels of reversible deformation reached $50 \%$. By the method of mathematical modeling, it is shown that the Poisson's ratio of super carbon nanotubes depends on the deformation processes occurring in them. Possessing a channel structure at the nanoscale, supercarbon nanotubes have a wide potential for use as nanotubes and nanomaterials.

Models of the mechanical behavior of nanoparticles, nanostructures, and composite objects of them are considered in [21]. We used methods for modeling solid mechanics for the study of nanocomposites and experimental approaches. The processes of formation of defects and nanomaterials reinforced by particles and nanoinclusions are given. The analysis of the possibility of calculating and estimating the effective deformation parameters of nanostructures and nanocomposites has been carried out. In this paper, nanofilms, nanotubes, thin-layer nanocoatings are considered as nanomaterials. The processes of deformation, fracture and formation of cracks are discussed taking into account the influence of the internal structure, the appearance and modification of defects, the size factor and the dislocation mechanisms of plastic deformation with respect to nanostructured materials.

The purpose of this work is to describe the theoretical foundations and mechanisms for modeling the deformation and fracture of nanocomposites. The work is a development and generalization of the earlier publications of the authors [2224], where the main attention is paid to the generalized macro characteristics of nanoparticles and nanocomposites based on them as a whole.

\section{THEORETICAL BASES OF THE MOLECULAR DYNAMICS METHOD}

he study of the behavior and properties of nanosystems and nanoobjects was carried out by the method of molecular dynamics. Molecular dynamics can accurately reproduce the basic properties and parameters of nanomaterials, but the results will strongly depend on the choice of the power potential responsible for the interaction of atoms in the system. In the work, the many-particle potential of MEAM (modified embedded atom method) was used for the problems to be solved. The mathematical model of molecular dynamics and MEAM potential is described in more detail in previously published papers [25-27].

The total potential energy of the nanosystem in MEAM is represented by the sum of the energies of individual atoms 


$$
U^{M E A M}(r)=\sum_{i} U_{i}(r)=\sum_{i}\left(F_{i}\left(\bar{\rho}_{i}\right)+\frac{1}{2} \sum_{j \neq i} \phi_{i j}\left(r_{i j}\right)\right), i=1,2, \ldots, N,
$$

where $U_{i}(r)$ - is the potential of the $i$-th atom, the potential affects the type of interaction of atoms and the magnitude of the forces in the equations of motion; $F_{i}$ - the immersion function of the $i$-th atom located at a point in space with electron background density $\bar{\rho}_{i} ; N$ - the number of elements of the nanosystem, atoms or nanoparticles; $\phi_{i j}\left(r_{i j}\right)-$ the value of the pair potential between the $i$-th and $j$-th atoms, remotely separated on $r_{i j}$.

The immersion function depends on the background electron density, has a variable form for different types of chemical elements of the periodic system and is written using the expression

$$
F_{i}\left(\bar{\rho}_{i}\right)=\left\{\begin{array}{l}
A_{i} E_{i}^{0}\left(\bar{\rho}_{i}\right) \ln \left(\bar{\rho}_{i}\right), \bar{\rho}_{i} \geq 0 \\
-A_{i} E_{i}^{0} \bar{\rho}_{i}, \bar{\rho}_{i}<0
\end{array}\right.
$$

where $A_{i}$ - is the empirical parameter of the potential field; $E_{i}^{0}$ - the value of the energy of sublimation; $\bar{\rho}_{i}-$ background electron density; the index $i$ indicates the ownership of a particular type of atom.

The background electron density at the point of immersion is determined by the following functional dependence

$$
\bar{\rho}_{i}=\frac{\rho_{i}^{(0)}}{\rho_{i}^{0}} G\left(\Gamma_{i}\right), \quad \Gamma_{i}=\sum_{k=1}^{3} t_{i}^{(k)}\left(\frac{\rho_{i}^{(k)}}{\rho_{i}^{(0)}}\right)^{2}
$$

where the indices $k=1,2,3$ correspond to the $\mathrm{p}, \mathrm{d}, \mathrm{f}$ electron orbitals of the $i$-th atom; $t_{i}^{(k)}$ - weight coefficients of the model; $\rho_{i}^{0}$ - background electron density of the initial structure; $\rho_{i}^{(k)}$ - parameters characterizing the deviation of the electron density from its ideal state, when all atoms are in the lattice sites.

Different formulations are used to calculate the function $G(\Gamma)$. The total background electron density $\bar{\rho}_{i}$ contains partial contributions of individual densities of atomic orbitals. Atomic orbitals are divided into spherically symmetric s, which corresponds to electron density $\rho_{i}^{(0)}$, and angular $\mathrm{p}, \mathrm{d}$, f clouds, with distributions $\rho_{i}^{(1)}, \rho_{i}^{(2)}, \rho_{i}^{(3)}$.

To determine the weights of the model of (3), the expression is used

$$
t_{i}^{(k)}=\frac{\sum_{i \neq j} t_{0, j}^{(k)} \rho_{j}^{A(0)} S_{i j}}{\sum_{i \neq j}\left(t_{0, j}^{(k)}\right)^{2} \rho_{j}^{A(0)} S_{i j}},
$$

where $t_{0, j}^{(k)}$ are the parameters depending on the chemical type of the $j$-th element.

Together with the MEAM potential, the shielding function is used, which is used to reduce the computational cost and reduce the potential error

$$
\begin{aligned}
& S_{i j}=f_{c}\left(\frac{r_{c}-r_{i j}}{\Delta r}\right) \prod_{k \neq i, j} f_{c}\left(\frac{C_{i k j}-C_{\text {min }, i k j}}{C_{\text {max }, i k j}-C_{\text {min }, i k j}}\right), \\
& C_{i k j}=1+2 \frac{r_{i j}^{2} r_{i k}^{2}+r_{i j}^{2} r_{j k}^{2}-r_{i j}^{4}}{r_{i j}^{4}-\left(r_{i k}^{2}-r_{j k}^{2}\right)^{2}},
\end{aligned}
$$




$$
f_{c}(x)=\left\{\begin{array}{l}
1, x \geq 1 \\
{\left[1-(1-x)^{4}\right]^{2}, 0<x<1} \\
0, x \leq 0
\end{array}\right.
$$

where $r_{c}$ - is the potential cutoff radius; $C_{\min }, C_{\max }$ - are formulated for each triple of atoms $i, j, k$ and depend on their chemical types; $\Delta r$-determines the distance exceeding the cutoff radius at which smooth smoothing of the force field occurs.

The theory of a modified immersed atom method is currently considered one of the most recognized approaches to the description of the electronic properties of nanomaterials and is used to study and predict the properties of metals, semiconductors and other objects of complex structure and dense packing of atoms.

As a result of the conducted numerical studies, detailed information about all atoms of the nanosystem at each moment of time becomes known. The underlying basic variables are the velocities and coordinates of the particles, as well as the forces acting between them. Based on the obtained variables, other parameters of nanostructures are calculated, macroparameters and mechanical characteristics are determined.

\section{CALCULATION OF STRESS TENSORS AND DEFORMATIONS}

7 o calculate the stress and strain tensors in nanosystems, several approaches are currently used. Methods for calculating these tensors have a different physical nature and rely on alternative mathematical tools. Consider two basic methods in more detail.

The first approach is based on the relationships of continuum mechanics [28] and provides for the construction of the calculation process in the "top - down" direction. At the preliminary stage of calculating the elastic characteristics, an elementary volume is allocated, in relation to which these characteristics will be related. There are certain difficulties with the calculation of the elementary volume in nanomaterials, since there is no unambiguous formulation of the volume of an individual atom. In addition, between the atoms in the system there are voids, inhomogeneities and dislocations, which also need to be included in the volume of the sample under study. Incorrect consideration of intercrystalline faces can lead to inaccurate and erroneous calculations of stress and strain tensors. On the one hand, an increase in the size of the sample makes it possible to reduce the magnitude of the error, on the other hand, based on the definitions of continuum mechanics, the volume should remain infinitely small.

Having determined the value of the elementary volume, calculate the area of the planes bounding it. For definiteness, we denote the area of $S_{\alpha \beta}$ borders as. Hereinafter, the indices $\alpha$ and $\beta$ indicate the direction and position of the boundary, for a three-dimensional Cartesian coordinate system $\alpha, \beta=\{1,2,3\}$. Some facets of the volume are applied forces $F_{\alpha \beta}$ that can act tangentially and normal, therefore, when designating forces, there are also indices $\alpha$ and $\beta$. Under the action of forces, the nano-volume begins to deform, its geometry changes.

The components of the stress tensor are calculated through the corresponding forces and areas of the boundaries of the nanomaterial

$$
\sigma_{\alpha \beta}=\frac{F_{\alpha \beta}}{S_{\alpha \beta}}
$$

In the absence of an intrinsic angular momentum of a continuous medium, as well as bulk and surface stress pairs, the stress tensor is symmetric, that is $\sigma_{\alpha \beta}=\sigma_{\beta \alpha}$, and has six independent components.

The vector of displacements of the elementary volume is determined through the averaged displacements of all atoms using the values of the initial and final coordinates in accordance with the ratio

$$
\mathbf{u}=\frac{1}{N} \sum_{k=1}^{N} \mathbf{u}_{k}=\frac{1}{N} \sum_{k=1}^{N}\left(\mathbf{r}_{k}-\mathbf{r}_{k}^{\prime}\right)=\frac{1}{N} \sum_{k=1}^{N} \Delta \mathbf{r}_{k}
$$


where $\mathbf{u}$ is the vector of displacements of the elementary volume; $\mathbf{u}_{k}$ - displacement vector for the $k$-th atom; $N$ - the number of atoms in the nanosystem; $\mathbf{r}_{k}$ and $\mathbf{r}_{k}^{\prime}-$ the radius vectors of each atom before and after the movement, respectively.

The strain tensor, which is responsible for changing the shape and rebuilding the size of a nanomaterial, is associated with displacements by the expression

$$
\varepsilon_{\alpha \beta}=\frac{1}{2}\left(\frac{\partial u_{\alpha}}{\partial r_{\beta}}+\frac{\partial u_{\beta}}{\partial r_{\alpha}}+\sum_{l} \frac{\partial u_{l}}{\partial r_{\alpha}} \frac{\partial u_{l}}{\partial r_{\beta}}\right) \text {, }
$$

where $\varepsilon_{\alpha \beta}$ are the components of the strain tensor; $u_{\alpha}, u_{\beta}, r_{\alpha}, r_{\beta}$ - vector components of displacement and vector radius. Based on the definition, the strain tensor is also a symmetric quantity.

For small displacements, the summation over the index $l$ neglects and uses the strain tensor in a simpler form

$$
\varepsilon_{\alpha \beta}=\frac{1}{2}\left(\frac{\partial u_{\alpha}}{\partial r_{\beta}}+\frac{\partial u_{\beta}}{\partial r_{\alpha}}\right) .
$$

The calculation of the partial derivatives in (11) and (12) involves a number of difficulties, since it is not possible to obtain these expressions explicitly. Derivatives are determined numerically, which affects the accuracy and adequacy of the resulting strain tensor.

Using the tensors of stress and strain, Hooke's law can be written and various elastic properties of a nanomaterial such as Young's modulus, Poisson's ratio, shear modulus, and volume expansion coefficient can be calculated. In generalized form, Hooke's law is written as

$$
\sigma_{\alpha \beta}=\sum_{k, l} C_{\alpha \beta k l} \cdot \varepsilon_{k l},
$$

where $C_{\alpha \beta k l}$ is the fourth rank tensor. For an isotropic material, the tensor $C_{\alpha \beta k l}$ contains only two independent coefficients. Using the first approach to calculate the elastic parameters of metal nanoparticles, the authors conducted research. These studies were previously published in $[10,11]$.

The second approach is an alternative, provides for the construction of the calculation process in the "bottom - up" direction and relies on the kinetic theory and the virial theorem [29, 30]. The virial theorem connects the average kinetic energy of a nanosystem with the average potential energy and allows us to take into account not only the component of the molecular-kinetic theory of an ideal gas, but also the influence of the properties of real atoms:

$$
P=\frac{N k_{B} T}{W}+\frac{1}{3 W} \sum_{k=1}^{N^{\prime}} \mathbf{r}_{k} \cdot \mathbf{F}_{k}
$$

where $W$ is the volume of the settlement area; $\mathbf{F}_{k}$ - the resultant force acting on the $k$-th atom; $\mathbf{r}_{k}-$ is the radius vector of the $k$-th atom. The value $N^{\prime}$ is responsible for the number of atoms, taking into account the symmetric images used in the simulation with periodic boundary conditions. The second term in (13) is virial.

The components of the nanosystem pressure tensor are calculated in a similar way. Given the relationship between the kinetic and potential energies and coordinatewise decomposition of the vectors, the expression for the pressure tensor is used

$$
P_{\alpha \beta}=\frac{1}{W} \sum_{k=1}^{N} m_{k} V_{k, \alpha} V_{k, \beta}+\frac{1}{W} \sum_{k=1}^{N^{\prime}} r_{k, \alpha} \cdot F_{k, \beta},
$$


where $m_{k}$ is the mass of the $k$-th atom; $V_{k, \alpha}, V_{k, \beta}$ - components of the velocity of the $k$-th atom; $r_{k, \alpha}, F_{k, \beta}$ - elements of the radius vector and force for the $k$-th atom, respectively.

Due to the dependence of the force on the potential gradient, the stress-strain state will ultimately also be determined by the potential field. For an individual atom under the number $k$, the analogue of the stress tensor is written as the following expression

$$
\begin{aligned}
& f_{k, \alpha \beta}=-\left[m_{k} V_{k, \alpha} V_{k, \beta}+\frac{1}{2} \sum_{n=1}^{N_{p}}\left(r_{1, \alpha} F_{1, \beta}+r_{2, \alpha} F_{2, \beta}\right)+\frac{1}{2} \sum_{n=1}^{N_{h}}\left(r_{1, \alpha} F_{1, \beta}+r_{2, \alpha} F_{2, \beta}\right)+\right. \\
& +\frac{1}{3} \sum_{n=1}^{N_{\alpha}}\left(r_{1, \alpha} F_{1, \beta}+r_{2, \alpha} F_{2, \beta}+r_{3, \alpha} F_{3, \beta}\right)+\frac{1}{4} \sum_{n=1}^{N_{d}}\left(r_{1, \alpha} F_{1, \beta}+r_{2, \alpha} F_{2, \beta}+r_{3, \alpha} F_{3, \beta}+r_{4, \alpha} F_{4, \beta}\right)+ \\
& \left.\frac{1}{4} \sum_{n=1}^{N_{i}}\left(r_{1, \alpha} F_{1, \beta}+r_{2, \alpha} F_{2, \beta}+r_{3, \alpha} F_{3, \beta}+r_{4, \alpha} F_{4, \beta}\right)+\operatorname{Kspace}\left(r_{k, \alpha}, F_{k, \beta}\right)+\sum_{n=1}^{N_{f}} r_{k, \alpha} F_{k, \beta}\right] .
\end{aligned}
$$

Formula (15) takes into account the different types of potentials and interactions and the kinetic energy of the atom. The first term is the contribution of kinetic energy to the force tensor; therefore, the product of the components of velocity is present in it. The second term of the sum is responsible for the pair interaction, where $N_{p}$ is the number of the nearest neighbors of the atom $k$ participating in the pair interaction, and $\mathbf{F}_{1}$ and $\mathbf{F}_{2}$ are the forces that arise. The third term corresponds to the nodal potential, therefore under the sum sign there are the products of three radius vectors and forces, $N_{a}$ - the number of angles. Similar to the previous elements, $N_{d}$ and $N_{i}$ - the number of dihedral and pseudo-doubleangled angles, the interaction is calculated by four atoms. The term Kspace describes the long-range Coulomb contribution of the potential, and the last term relates to fixed and bounded particles, $N_{f}-$ the number of fixed atoms. In most problems, not all types of interactions are present, therefore, Eqn. (15) is significantly simplified.

For an infinitely small volume, the stress tensor is calculated by summing over all atoms

$$
\sigma_{\alpha \beta}=\frac{1}{W_{e}} \sum_{k=1}^{N} f_{k, \alpha \beta}
$$

where $W_{e}$ - the value of the elementary volume.

Displacements in the second approach are also determined on the basis of formula (9). On the basis of the distortion of the geometry of the elementary volume, for example, during the transition from a parallelepiped to a prismatic form, the strain tensor and the other mechanical parameters of the nanomaterial are found. In this paper, the second approach was used to describe the stress-strain state.

\section{RESULTS AND DISCUSSION}

I $\mathrm{n}$ the work, using the mathematical modeling by the method of molecular dynamics, the processes of deformation and failure of three types of materials are investigated: pure aluminum, a composite with an aluminum matrix and a filler in the form of spherical iron particles, and a composite with an aluminum matrix and a filler in the form of a cylindrical iron fiber. The simulation was performed on a cell with periodic boundary conditions on all its faces. Fig. 1 shows the coordinate system, computational cells and cross-sectional images along the middle of the cell along the $x$ axis of the composite with an aluminum matrix and filler in the form of spherical iron particles (a), as well as a composite with an aluminum matrix and filler in the form of cylindrical iron fiber (b). The dimensions of the computational cell for all calculations were along the $x$ axis $-11.4 \mathrm{~nm}$, and along the $y$ and $z$ axes $-5 \mathrm{~nm}$.

The physical dimensions of the calculated cell were the same for all calculations. The type of the atomic structure of the matrix and filler at the initial moment of time corresponded to the crystalline state of aluminum and iron. The geometry and dimensions of the nanocomposite fillers were as follows: the diameter of iron nanoparticles was $2 \mathrm{~nm}$, the crosssectional diameter of iron nanofibers was $2 \mathrm{~nm}$. The nanoparticle was placed in the center of the computational cell. The 
nanofibers were located in the center of the matrix along the $x$ axis throughout the computational cell. The weight content of the filler was: for nanoparticles $4.23 \%$, for nanofibers $28.42 \%$. The use of periodic boundary conditions made it possible to model the deformation and fracture of an "infinite" sample of a nanocomposite formed by the calculation cells along all coordinate axes.

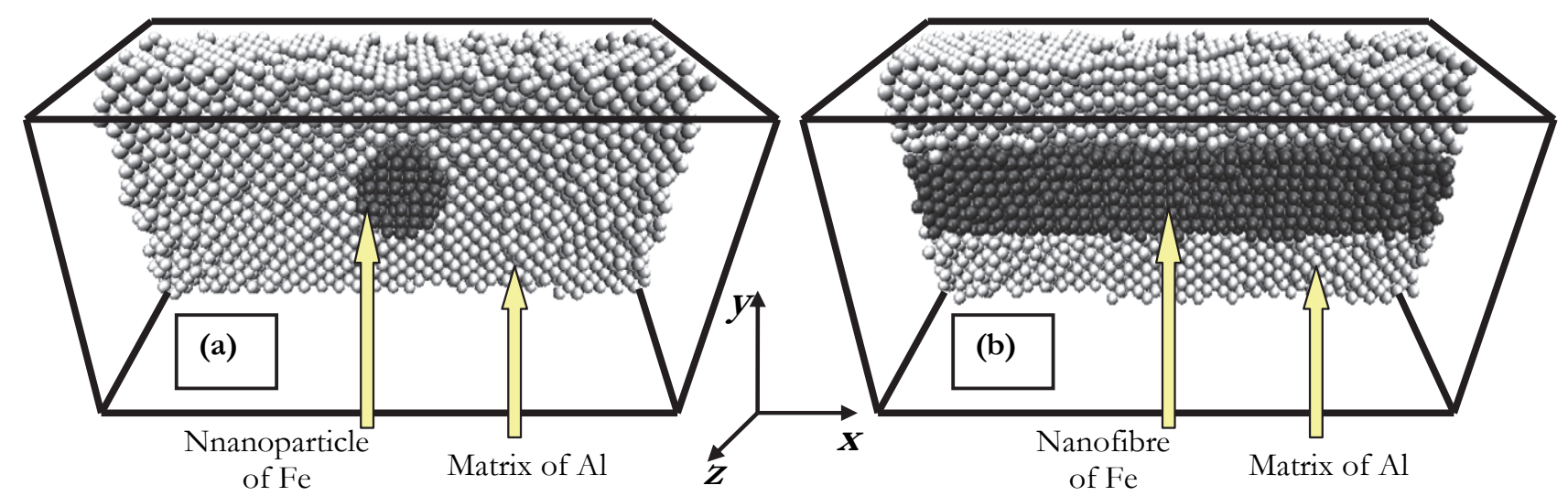

Figure 1: Cross sections of nanocomposite samples with an aluminum matrix and a spherical nanoparticle of aluminum iron (a) and iron nanofibers (b), after relaxation to the deformation stage.

After placing the filler (nanoparticles or nanofibers of iron atoms) into a computational cell, which already contains aluminum atoms, the atomic system was relaxed for 20 picoseconds. Relaxation was the restructuring of the atomic system under constant thermodynamic conditions at a temperature of $300 \mathrm{~K}$ and the absence of external loads. The temperature was kept constant using the Nose-Hoover thermostat described in detail in article [31]. Relaxation was performed to relieve residual stresses and stabilize the nanosystem.

For pure aluminum, the sample was also relaxed for 20 picoseconds under normal thermodynamic conditions. The temperature was also maintained at $300 \mathrm{~K}$. Graphic results for pure aluminum are not shown in Fig. 1, since the structure of the sample did not change during the relaxation process.

When modeling the deformation and failure of a nanocomposite, two types of deformation were investigated. In the first case, the sample was subjected to uniform stretching along the $x$ axis; in the second variant, shear strain was studied in the $x y$ plane. The deformation patterns are shown in Fig. 2.
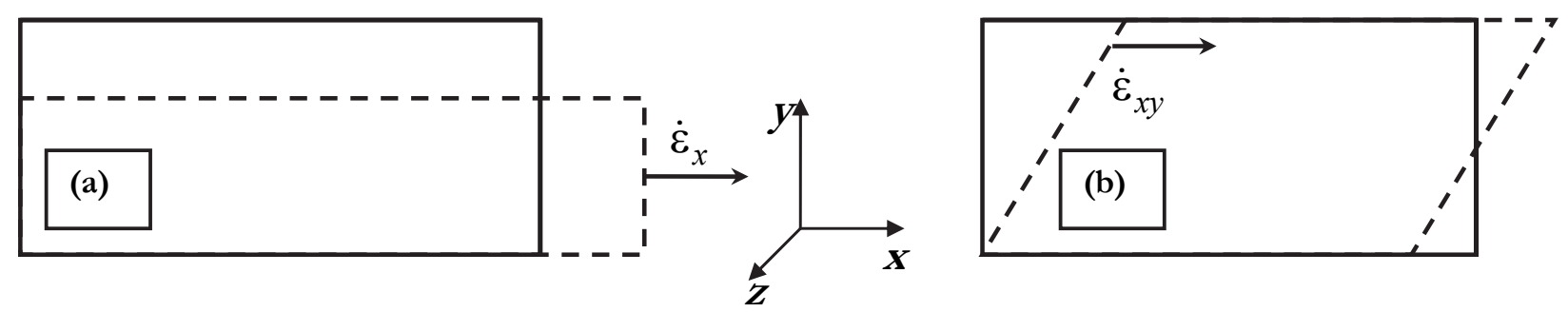

Figure 2: Nanocomposite loading scheme for the uniaxial tension (a) and for shear deformation (b).

The deformation of the sample was carried out as follows. At each time step, the nanosystem calculation cell changed its geometry. To stretch the nanocomposite, the computational cell expanded along the $x$ axis at a constant speed. For shear deformation, the computational cell changed its geometry, and the original sample in the form of a rectangular parallelepiped turned into a prism. The change in the computational domain is associated with the recalculation of the coordinates of all atoms of the nanosystem. Thus, after cell deformation, all atoms changed their positions in proportion to the magnitude with which the computational domain is modified. After changing the coordinates of the atoms, the forces and velocities of the atoms acting on them were recalculated. In accordance with the current interatomic interaction potentials and the kinetics of motion, the atoms moved in one step in time. The action of the Nose-Hoover thermostat was carried out every 100-1000 time steps of integration. After the finally obtained velocities, coordinates, and forces acting in the atomic system, the parameters of the system were determined: the displacement of atoms of the nanosystem, the stress and strain tensors. 
Let us consider in detail the picture of the deformation and failure of the material in the process of stretching and shear deformation. The deformation processes of tension and failure of the specimen from pure aluminum and nanocomposites under study are shown in Figs. 3, 4 and 5, respectively. Tension patterns are presented for various moments of modeling.
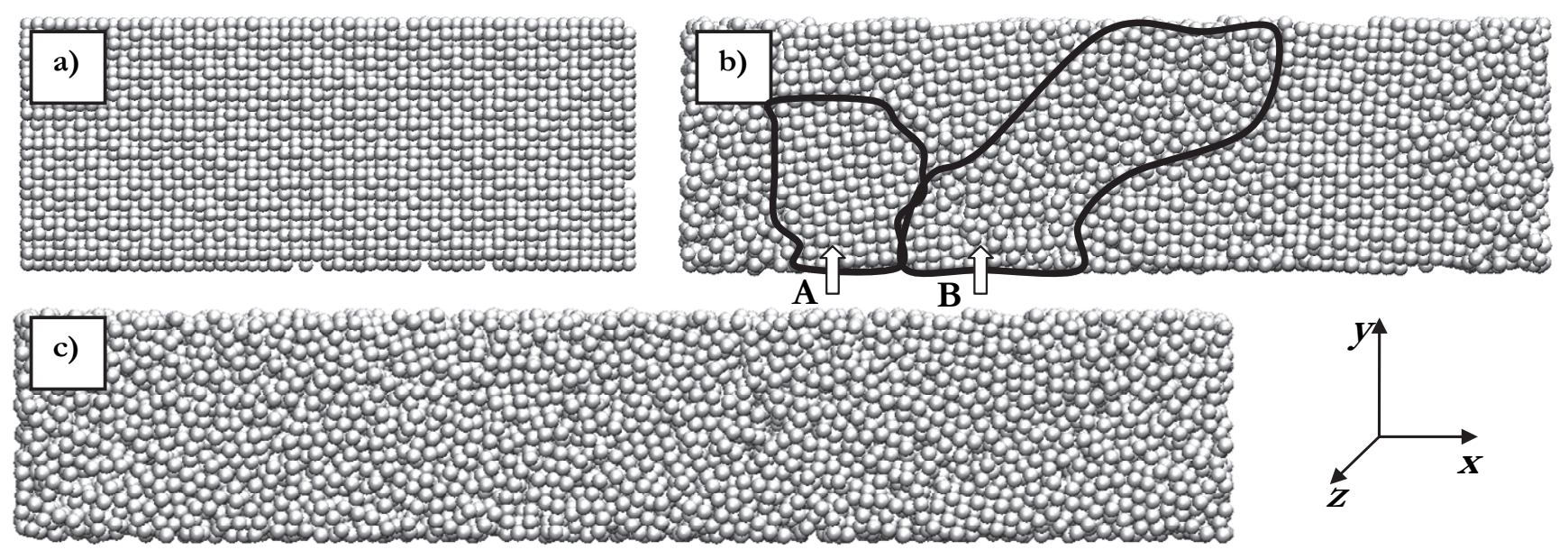

Figure 3: Pure aluminum sample tensile strain for simulation time of 5 psec (deformation 0.05) a; 33 psec (deformation 0.33 ) b; 80 psec (deformation 0.80) c.

The images in Fig. 3 illustrate the stretching of pure aluminum. Note that, within a small strain along the $x$-axis to 0.05 , the structure of the material remains almost unchanged (Fig. 3 a). Then, as deformation increases, an active restructuring of the atomic structure of the material follows. Atoms are displaced relative to the lattice sites (Fig. $3 \mathrm{~b}$ ). In the structure of the material formed areas of defects (dislocations). Fig. $3 \mathrm{~b}$, corresponding to a strain of 0.33 , shows an example of such a region (B). In it, the symmetry of the coordinate positions of atoms is broken and new forms of equilibrium of atomic structure appear. At the same time, the crystallite regions (A) are preserved in the material. Further, with an increase in the deformation to 0.8 (Fig. $3 \mathrm{c}$ ), the area with defects fills the entire volume of the sample, its stretching is observed, the cross section decreases. Full rupture and failure at this stage does not occur, but the crystal structure of the material is completely transformed into amorphous.

The introduction of iron nanoparticles into the aluminum matrix dramatically changes the processes of deformation and failure of the material under tension. The patterns of deformation and rupture of a material with a nanoparticle are shown in Fig. 4. With small deformations along the $x$-axis to 0.05 , the structure of the material remains almost unchanged (Fig. $4 \mathrm{a}$ ) as in pure aluminum. Then, as the strain increases to 0.2 , a substantial rearrangement of the crystal lattice of aluminum atoms is observed (Fig. 4 b) only in the contact area of the matrix and the filler. The aluminum crystal lattice is distorted, and defects are formed in it. At the same time, the iron atoms forming the nanoparticle change their coordinates insignificantly, therefore the atomic structure of the nanoparticle remains almost unchanged. This is due to the stronger potential of interaction between iron atoms than between aluminum atoms. Note that the structure of the aluminum matrix that is not in contact with the nanoparticle remains unchanged.

The failure of an aluminum nanocomposite with an iron nanoparticle begins directly in the region of contact between the iron nanoparticle and the aluminum matrix as a result of the detachment of the aluminum matrix from the nanoparticle surface and subsequent rupture of the material (Fig. $4 \mathrm{c}$ ). Patterns of the nanocomposite structure with spherical inclusions shown in Fig. 4 b) and Fig. 4 c) correspond to different stages of failure. Fig. 4 b) illustrates the beginning of the process of irreversible deformation. The simulation was carried out using the molecular dynamics apparatus; therefore, any macroscopic criterion for describing the failure of a nanocomposite, the process of which is shown in Fig. 4 b) and Fig. 4 c), in the work was not used. In this case, the failure of the material determines the parameters of the potential of interaction of the atoms forming the nanocomposite. The failure of a nanocomposite occurs when atoms are removed to a distance at which the force of their interaction is zero. In the process loaded with an increasing number of atoms cease to interact with each other, forming separate local areas of failure, which with increasing lead to the complete destruction of the simulated material.

The deformation and failure of a nanocomposite with an aluminum matrix and an iron nanofiber for different levels of deformation is shown in Fig. 5. As in previous cases, with small deformations along the $x$-axis up to 0.05 , the structure of the matrix material and fiber practically does not change (Fig. 5 a). Then, as the strain increases to 0.2 , a substantial rearrangement of the crystal lattice is observed in a matrix of aluminum atoms and in some regions of the iron lattice (Fig. 
$5 \mathrm{~b})$. In places where the crystal lattice changes both in the matrix and in the nanofibers, defects are formed. With an increase in the strain to 0.8 (Fig. $5 \mathrm{c}$ ), the area with defects fills the entire volume of the matrix and almost the entire volume of nanofibers in which failure occurs.
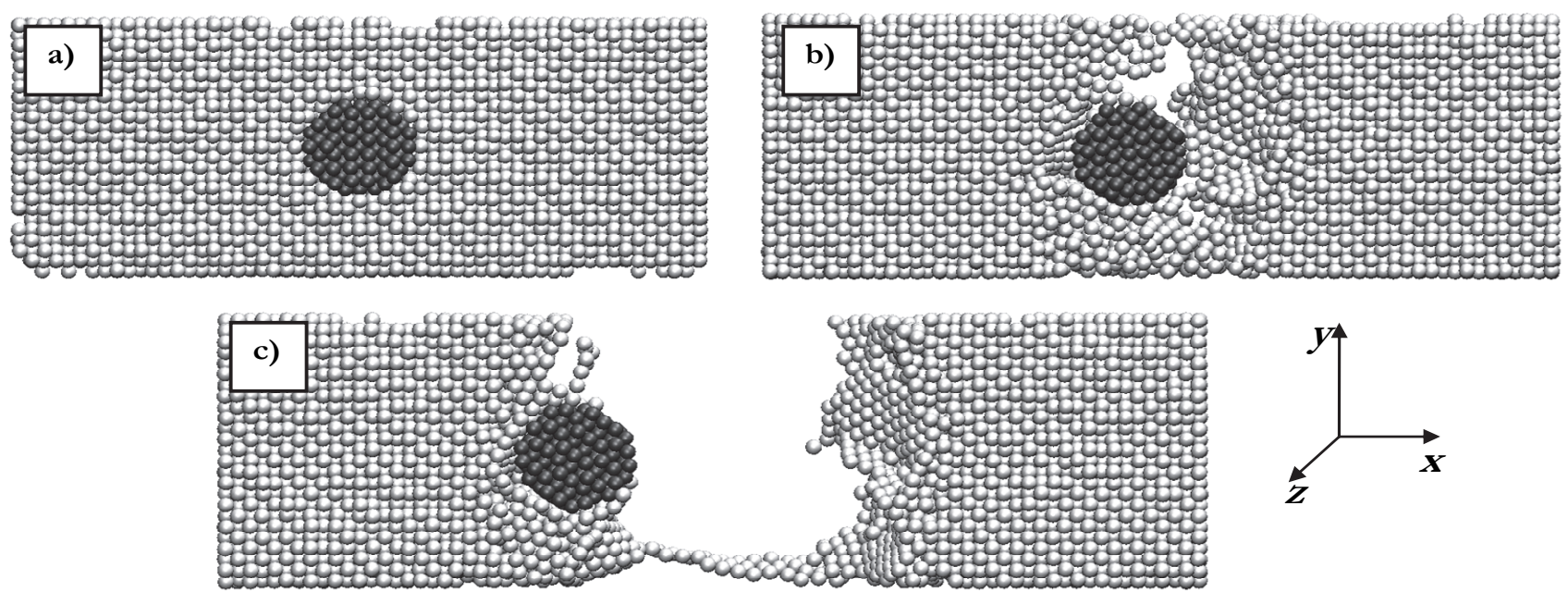

Figure 4: Tension deformation of an aluminum nanocomposite with an iron nanoparticle for a simulation time of 5 psec (deformation 0.05); 20 psec (deformation 0.20) b; 45 psec (deformation 0.45).
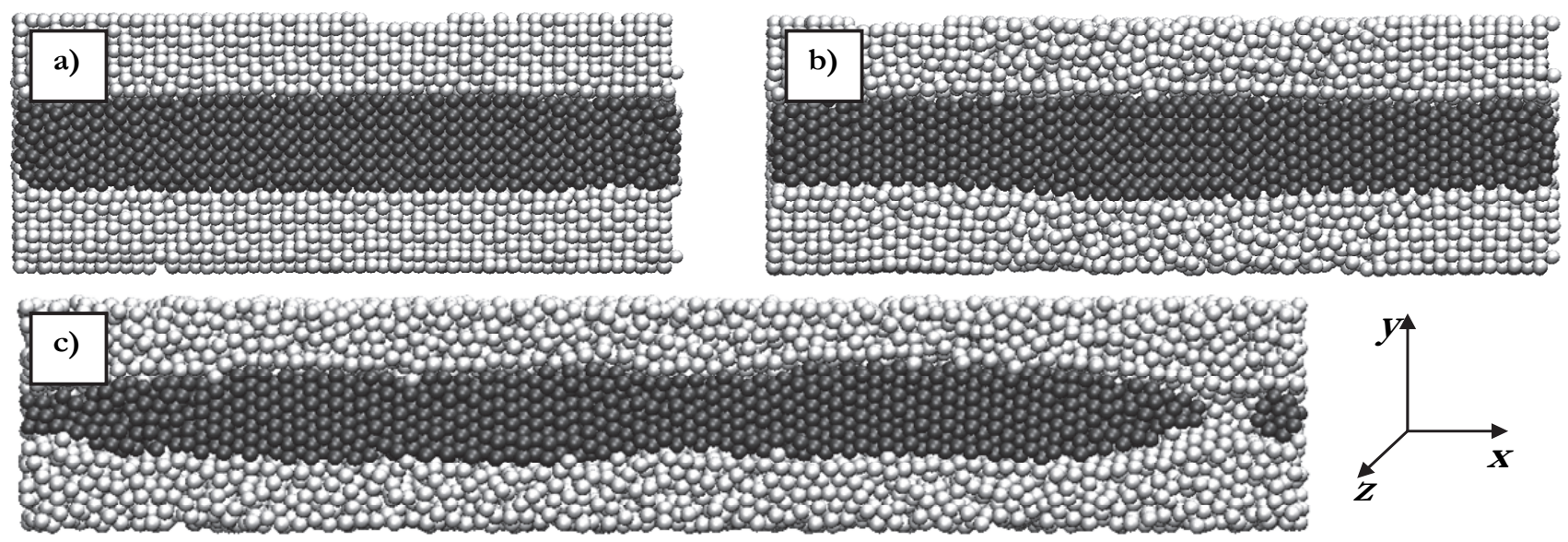

Figure 5: Tension deformation of an aluminum nanocomposite with an iron nanocylinder for a simulation time of 5 psec (deformation 0.05); 20 psec (deformation 0.20) b; 80 psec (deformation 0.80) c.

Consider the deformation and failure of the material during the deformation of pure shear. The processes of deformation and failure of aluminum and nanocomposites with nanoparticles and iron nanofibers for cases of shear deformation are shown in Figs. 6, 7, and 8, respectively. The shift of the computational domain was carried out in the $x y$ plane in the remaining planes the dimensional and angular parameters of the nanosystem remained unchanged.

First consider the deformation and failure of pure aluminum (Fig. 6). As in the previous examples, at small shear deformations up to 0.035 , the defects of the crystal structure in the sample is practically not observed (Fig. 6). In the process of deformation at this stage, the atoms have time to rearrange themselves and take a more energetically stable position. With a greater shear strain of 0.2 (Fig. 6 b), well observable straight shear bands of the crystal structure appear in the material, corresponding to plastic shear strain, often observed in experiments.

With a larger shear strain of more than 0.5 (Fig. 6 c), the shear bands lose stability and lattice defects develop. Formed areas of the amorphous state of aluminum, where the crystal structure is broken. There are also local areas with a preserved crystal lattice of various orientations and orientations. The central region of the sample is subjected to the greatest restructuring of the structure and the displacement of atoms relative to the lattice sites. 

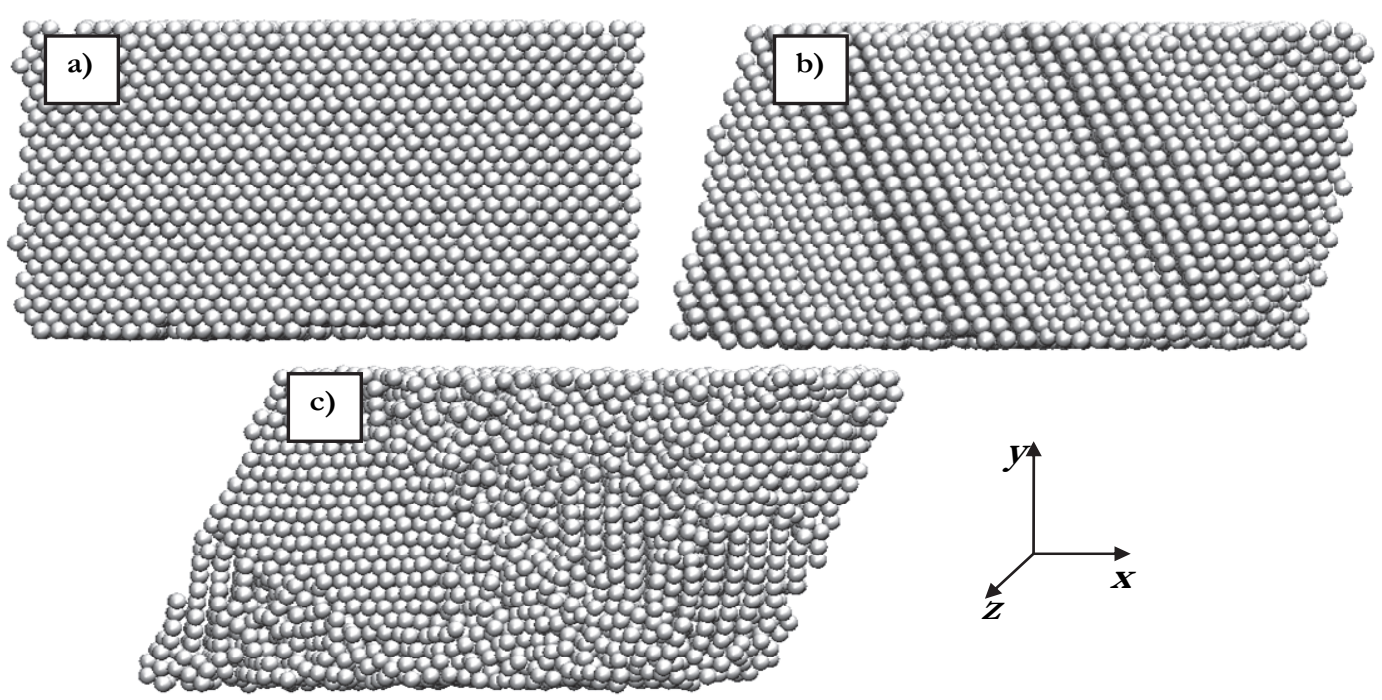

Figure 6: Pure aluminum sample shear strain for simulation time of 5 psec (deformation 0.035) a; 45 psec (deformation 0.315 ) b; 100 psec (deformation 0.700) c.
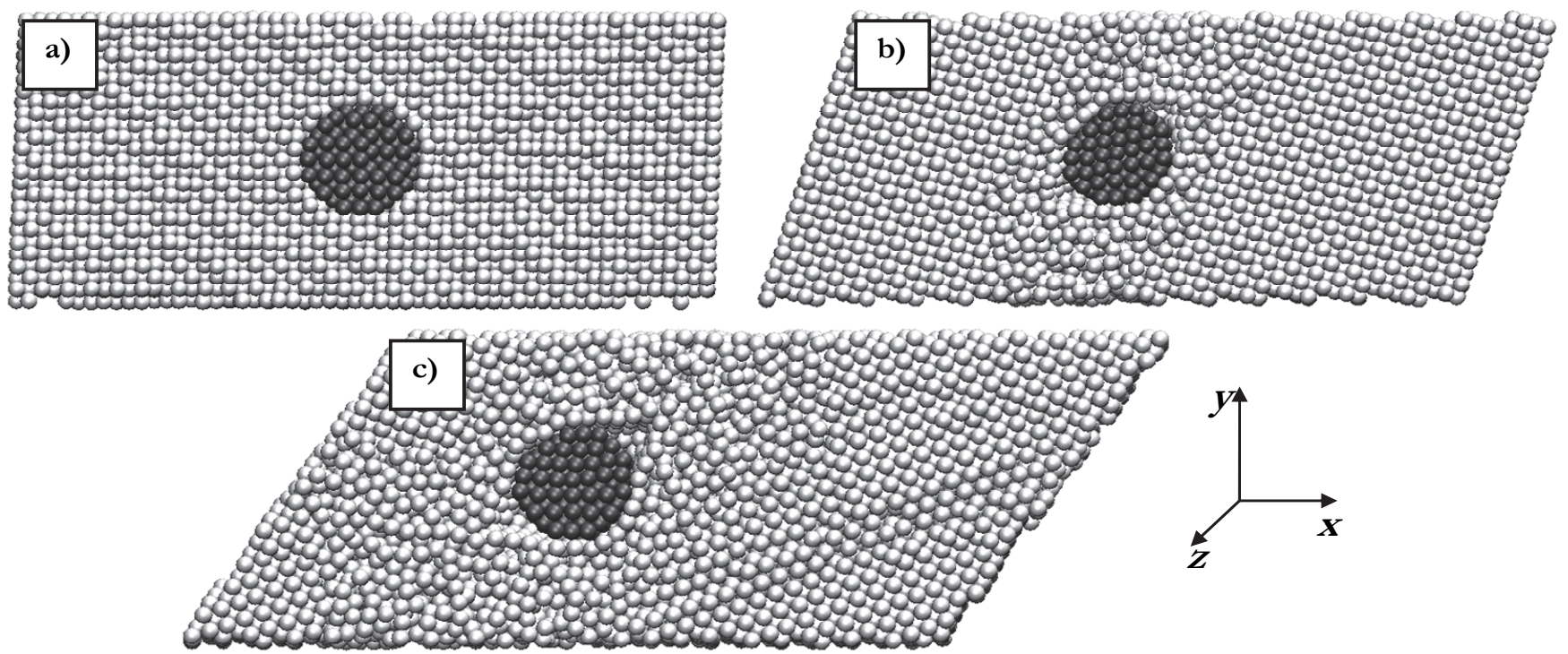

Figure 7: Shear deformation of an aluminum nanocomposite with an iron nanoparticle for a simulation time of 5 psec (deformation 0.035 ) a; 45 psec (deformation 0.315) b; 100 psec (deformation 0.700) c.

Shear strain patterns for a nanocomposite from an aluminum matrix with a spherical iron nanoparticle are shown in Fig. 7. For a strain of 0.035 (Fig. 7 a), there is no significant distortion of the sample structure. As the strain increases to 0.315 (Fig. 7 b), lattice distortions begin to nucleate near the iron nanoparticle. With this shear deformation in Fig. 7 b, the central layer with chaotically displaced aluminum atoms is clearly visible. This area of the amorphous state of the material with increasing deformation to 0.7 gradually spreads over the entire aluminum matrix, as can be seen from

Fig. 7 c. The structure of the nanoparticle remains crystalline and practically does not change during the shift. The failure of an aluminum nanocomposite with an iron nanoparticle during shear begins directly in the region of contact between the iron nanoparticle and the aluminum matrix as a result of the detachment of the aluminum matrix from the nanoparticle surface due to the mutual shear of the contacting surfaces of the nanoparticle and matrix.

An interesting result was obtained by studying the shear strain for a nanocomposite from an aluminum matrix and a nanofiber from iron. The deformation patterns of this material are shown in Fig. 8. In the process of increasing the shear strain from 0.035 (Fig. 8 a) to 0.315 (Fig. 8 b) and to 0.7 (Fig. 8 c), the changes in the crystal structure of the nanocomposite are insignificant. The amorphous phase is practically absent and no damage to the material is found in the 
investigated range of deformations. In this case, the nanofiber of iron atoms is a kind of reinforcing material. Nanofiber smooths the main stresses and strains. The rearrangement of the coordinate positions of the atoms of the nanocomposite proceeds gradually. Thus, this nanocomposite is more resistant to damage during shear deformation than a nanocomposite with a nanoparticle.
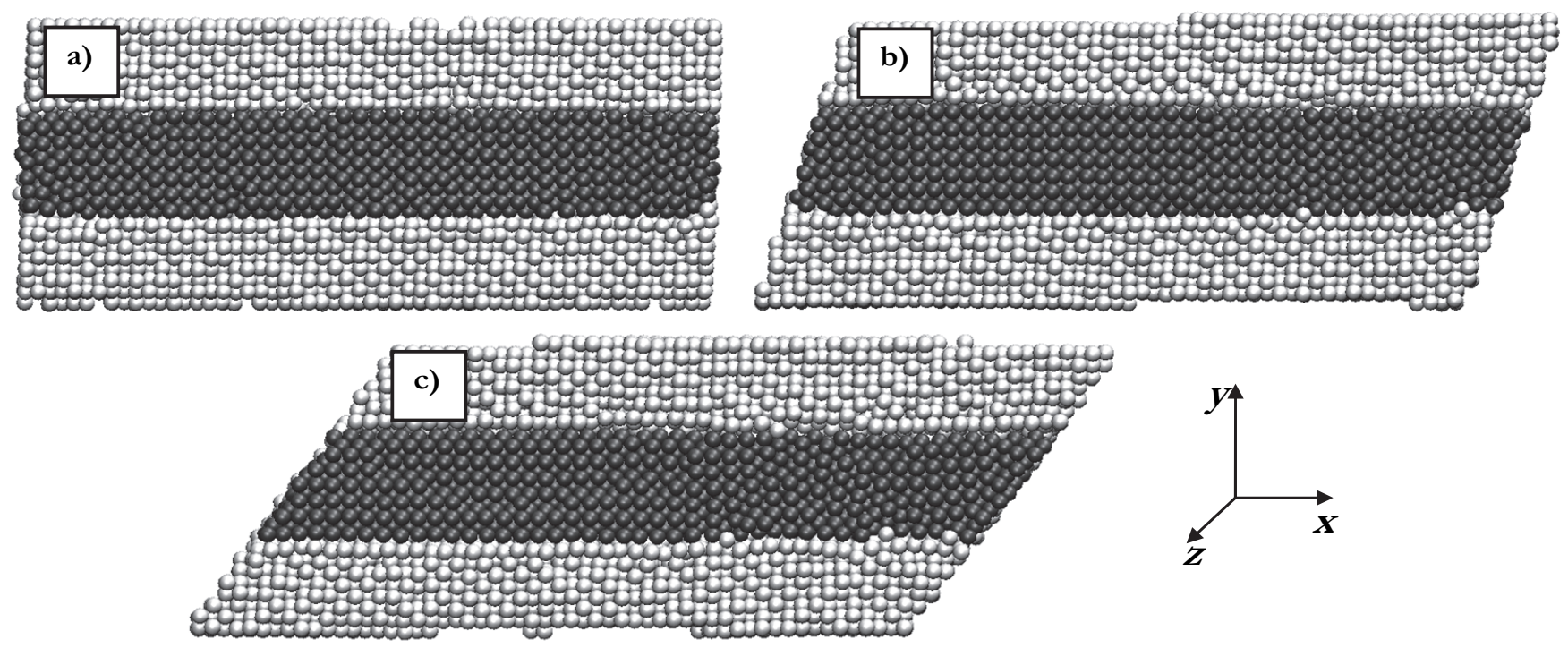

Figure 8: Shear deformation of an aluminum nanocomposite with an iron nanocylinder for a simulation time of 5 psec (deformation 0.035) a; 45 psec (deformation 0.315) b; 100 psec (deformation 0.700) c.

\section{CONCLUSIONS}

7 he paper proposes approaches to observe the processes of deformation and fracture of nanocomposites. Simulation of the processes of deformation and failure of nanocomposites was carried out by the method of molecular dynamics using the potential of the modified embedded atom method. The used multiparticle interatomic potential allows to study the behavior of composite materials under mechanical loading in detail at the nanoscale level, analyze the processes of deformation and fracture, and study the nature of the origin and development of defects and dislocations in them.

The results of the research can be used to study the processes of deformation of nanocomposite materials with promising functional properties. The tasks of further research include studies of nanocomposites with a more complex structure and complex deformation programs.

\section{ACKNOWLEDGMENTS}

7 he work was supported by IzSTU (project 28.04.01 / 18VAV), Research Program of the Ural Branch of the Russian Academy of Sciences (project 18-10-1-29) and UFRC (project 0427-2019-0029).

\section{REFERENCES}

[1] Uemura, T., Kitagawa, S. (2005). Nanocrystals of Coordination Polymers, Chemistry Letters, 34(2), pp. 132-137. DOI: $10.1246 / \mathrm{cl} .2005 .132$.

[2] Kogai, V.Ya., Vakhrushev, A.V., Fedotov, A.Yu. (2012). Spontaneous Explosive Crystallization and Phase Transformations in a Selenium/Copper Bilayer Nanofilm, Journal of Experimental and Theoretical Physics Letters, 95(9), pp. 454-456. DOI: 10.1134/S0021364012090068. 
[3] Wang, L., Qi, T., Zhang, Y. (2005). Synthesis, Characterization, and Boron Uptake of Organic-Inorganic Hybrid Mesoporous Materials, Chemistry Letters, 34(2), pp. 144-145. DOI: 10.1246/cl.2005.144.

[4] Srinivas, K., Rao, D.K. (2014). Modelling and Computer Simulation of Nanostructured Devices, American Journal of Nanoscience and Nanotechnology, 2(3), pp. 40-44. DOI: 10.11648/j.nano.20140203.12.

[5] Han, S., Lee, M.H., Ihm, J. (2002). Dynamical Simulation of Field Emission in Nanostructures, Physical Review B, 65, pp. 085405.1-7. DOI: 10.1103/PhysRevB.65.085405.

[6] Grishanov, E.N., Popov, I.Y. (2016). Computer Simulation of Periodic, Nanostructures. Nanosystems: Phisics, Cnemistry, Mathematics, 7(5), pp. 865-868. DOI: 10.17586/2220-8054-2016-7-5-865-868.

[7] Vakhrushev, A.V., Fedotov, A.Yu. (2014). Multilevel mathematical modeling of condensation processes in aerosol nanosystems, International Scientific Journal Alternative Energy and Ecology, 8, pp. 8-21.

[8] Severyukhin, A.V., Severyukhina, O.Yu., Vakhrushev, A.V., Fedotov, A.Yu. (2016). Investigation of thermophysical properties of silicon nanomaterials by Green-Kubo method. Proceedings of the Institute of Mechanics, Ural Branch of the Russian Academy of Sciences "Problems of Mechanics and Materials Science", pp. 210-223.

[9] Turko, B.I., Kapustianyk, V.B., Rudyk, V.P., Rudyk, Y.V. (2016). Thermal Conductivity of Zinc Oxide Micro- and Nanocomposites, Journal of Nano- and Electronic Physics, 8(2), pp. 02004.1-4. DOI: 10.21272/jnep.8(2).02004.

[10] Wen, T., Brush, L.N., Krishnan, K.M. (2014). A Generalized Diffusion Model for Growth of Nanoparticles Synthesized by Colloidal Methods, Journal of Colloid and Interface Science, 419, pp. 79-85. DOI: $10.1016 /$ j.jcis.2013.12.018.

[11] Lechner, M.D., Mächtle, W. (2011). Characterization of Nanoparticles, Macromolecular Symposia, 145(1), pp. 1-7. DOI: $10.1002 /$ masy.19991450103.

[12] Smitha, S., Sango, S.L., Asaithambi, T. (2014-2015). Electrical Conductivity and Band Gap Energy Study of Some Transparent Conducting Metal Oxide and Sulphide Nano Materials, Int. J. ChemTech Res., 7(5), pp $2133-2137$.

[13] Dirmyer, M.R., Martin, J., Nolas, G.S., Sen, A., Badding, J.V. (2009). Thermal and Electrical Conductivity of SizeTuned Bismuth Telluride Nanoparticles, Small, 5(8), pp. 933-937. DOI: 10.1002/smll.200801206.

[14] Vakhrushev, A.V., Fedotov, A.Yu., Shushkov, A.A., Shushkov, A.V. (2011). Modeling of the Formation of Metal Nanoparticles, Study of Structural, Physical-Mechanical Properties of Nanoparticles and Nanocomposites, News of Tula State University. Natural Sciences, Physical sciences, 2, pp. 241-253.

[15] Vakhrushev, A.V., Fedotov, A.Yu., Vakhrushev, A.A., Shushkov, A.A., Shushkov, A.V. (2010). The Study of Mechanisms of Formation of Metal Nanoparticles, Determination of Mechanical and Structural Characteristics of Nano-objects and Composite Materials on its Basis, Chemical Physics and Mesoscopics,12(4), pp. $486-495$.

[16] Ribarsky, M., Landman, U. (1988). Dynamical Simulation of Stress, Strain and Finite Deformation, Phys. Rev. B, 38(14), pp. 9522-9537. DOI: 10.1103/PhysRevB.38.9522.

[17] Lee, S W, Cheng, Y T, Ryu, I, et al. (2014). Cold-temperature deformation of nano-sized tungsten and niobium as revealed by in-situ nano-mechanical experiments, Sci China Tech Sci, 57, pp. 652-662. DOI: $10.1007 /$ s11431-014-5502-8.

[18] Begue, F., Pujol, P., Ramazashvili, R. (2018). Identifying Two-Dimensional Z2 Antiferromagnetic Topological Insulators, Journal of Experimental and Theoretical Physics, 153(1), pp. 108-126. DOI: $10.7868 /$ S0044451018010108.

[19] Gutkin, M.Yu., Ovid'ko, I.A., Pande, C.S. (2001). Theoretical models of plastic deformation processes in nanocrystalline materials, Rev. Adv. Mater. Sci., 2, pp. 80-102.

[20] Shi, X., He, X., Wang, L., Sun, L. (2017). Hierarchical-structure induced adjustable deformation of super carbon nanotubes with radial shrinkage up to 66\%, Carbon, 125, pp. 289-298. DOI: 10.1016/j.carbon.2017.09.053.

[21] Goldstein, R.V., Morozov, N.F. (2007). Mechanics of deformation and fracture of nanomaterials and nanotechnology, Physical Mesomechanics, 10(5-6), pp. 235-246. DOI: 10.1016/j.physme.2007.11.002.

[22] Vakhrushev, A.V, Fedotov, A.Yu. (2008). Probabilistic analysis of modeling the distribution of structural characteristics of composite nanoparticles formed in the gas phase, Computational Continuum Mechanics, 1(3), pp. 34-45. DOI: 10.7242/1999-6691/2008.1.3.25.

[23] Vakhrushev, A.V., Fedotov, A.Y., Vakhrushev, A.A., Golubchikov, V.B., Givotkov, A.V. (2011). Multilevel Simulation of the Processes of Nanoaerosol Formation. Part 2. Numerical Investigation of the Processes of Nanoaerosol Formation for Suppression of Fires, International Journal of Nanomechanics Science and Technology, 2(3), pp. 205-216. DOI: 10.1615/NanomechanicsSciTechnolIntJ.v2.i3.20.

[24] Vakhrushev, A.V., Fedotov, A.Y., Vakhrushev, A.A. (2011). Modeling of Processes of Composite Nanoparticle Formation by the Molecular Dynamics Technique. Part 1. Structure of Composite Nanoparticles, Journal of Nanomechanics Science and Technology, 2(1), pp. 9-38. DOI: 10.1615/NanomechanicsSciTechnolIntJ.v2.i1.20. 
[25] Vakhrushev, A.V., Fedotov, A.Yu., Severyukhin, A.V., Suvorov, S.V. (2014). Modelling of Processes of Special Nanostructured Layers of Epitaxial Structures for Sophisticated Photovoltaic Cells, Chemical Physics and Mesoscopics, 16(3), pp. 364-380.

[26] Vakhrushev, A.V., Fedotov, A.Yu., Severyukhin, A.V., Valeev, R.G. (2015). Simulation of deposition processes of nanofilms on a porous alumina substrate, Chemical Physics and Mesoscopics, 17(4), pp. 511-522.

[27] Vakhrushev, A.V., Fedotov, A.Yu., Severyukhin, A.V., Valeev R.G. (2016). Investigation of deposition of nanofilms on a substrate of porous alumina using mathematical modeling, Computational Continuum Mechanics, 9(1), pp. 59_ 72. DOI: 10.7242/1999-6691/2016.9.1.6.

[28] Golovneva, E.I., Golovnev, I.F., Fomin, V.M. (2005). Peculiarities of application of continuum mechanics methods to the description of nanostructures, Phys. Mesomech., 8(5-6), pp. 41-48.

[29] Hummer, G., Gronbech-Jensen, N., Neumann, M. (1998). Pressure Calculation in Polar and Charged Systems Using Ewald Summation: Results for the Extended Simple Point Charge Model of Water, Journal of Chemical Physics, 109(7), pp. 2791.1-19. DOI: 10.1063/1.476834.

[30] Thompson, A.P., Plimpton, S.J., Mattson, W. (2009). General Formulation of Pressure and Stress Tensor for Arbitrary Many-Body Interaction Potentials under Periodic Boundary Conditions, J. Chem Phys, 131(15), pp. 154107.1-6. DOI: $10.1063 / 1.3245303$.

[31] Hoover, W. (1985). Canonical Dynamics: Equilibrium Phase-Space Distributions, Physical Review A, 31(3), pp. 16951697. DOI: $10.1103 /$ PhysRevA.31.1695. 